\title{
AXEL : A high pressure Xe gas TPC for neutrinoless double beta decay search
}

\author{
Sei BAN, ${ }^{* \dagger}$ \\ Kyoto University \\ E-mail: bansei0526escphys.kyoto-u.ac.ip
}

\begin{abstract}
AXEL is a high pressure xenon gas TPC detector which is under development for neutrinoless double beta decay search. We use the proportional scintillation mode with a new electroluminescence light detection scheme using Multi-pixel photon counter(MPPC) to achieve very high energy resolution with a large detector. The detector also has a capability of tracking and can reduce background. The project is in a R\&D phase. We report a demonstration of the concept of AXEL with $10 \mathrm{~L}$ and 4 bar prototype chamber. Some studies of basic property of MPPC are also reported.
\end{abstract}

\footnotetext{
* Speaker.

${ }^{\dagger}$ A.K.Ichikawa, T.Nakaya, A.Minamino, K.Nakamura, S.Yanagita, Y.Ishiyama, K.Haneda, M.Hirose, H.Sekiya, K.Ueshima, K.Miuchi
} 


\section{Introduction}

AXEL is a high pressure xenon gas time projection chamber (TPC), currently under development, for the search for neutrinoless double beta decay ( $0 v \beta \beta$ decay). This detector has three excellent features for $0 v \beta \beta$ decay search : extendable to large mass (1 ton enriched high pressure ${ }^{136} \mathrm{Xe}$ gas), good energy resolution (aiming $0.5 \% \mathrm{FWHM}$ at $2.48 \mathrm{MeV}$ which is $\mathrm{Q}$ value of ${ }^{136} \mathrm{Xe}$ ) and background rejection using event topological information. With these features, we are aiming to explore all the region of inverted hierarchy of neutrino masses.

Overview of the AXEL detector is illustrated in Fig. $\mathbf{m}$. Primary scintillation lights are detected by PMTs and used as the start timing of the event. Ionized electrons drift toward so called ElectroLuminescence Collection Cell (ELCC) plane and are converted to light signal by electroluminescence process. The concept of ELCC is shown in Fig. \. It consists of an anode plate, supporting PTFE plate, mesh and Multi-pixel photon counter(MPPC). The anode plate and PTFE plate have a few $\mathrm{mm}$ pitch holes. By applying high voltage between the anode plate and mesh, ionization electrons are collected into cells along the lines of electric field, and generate EL photons, which are detected by UV-sensitive MPPCs cell by cell. Because the EL region is contained in the each cell, this detector has less dependence on event position. Furthermore, It is easy to extend to large area due to the solid structure.
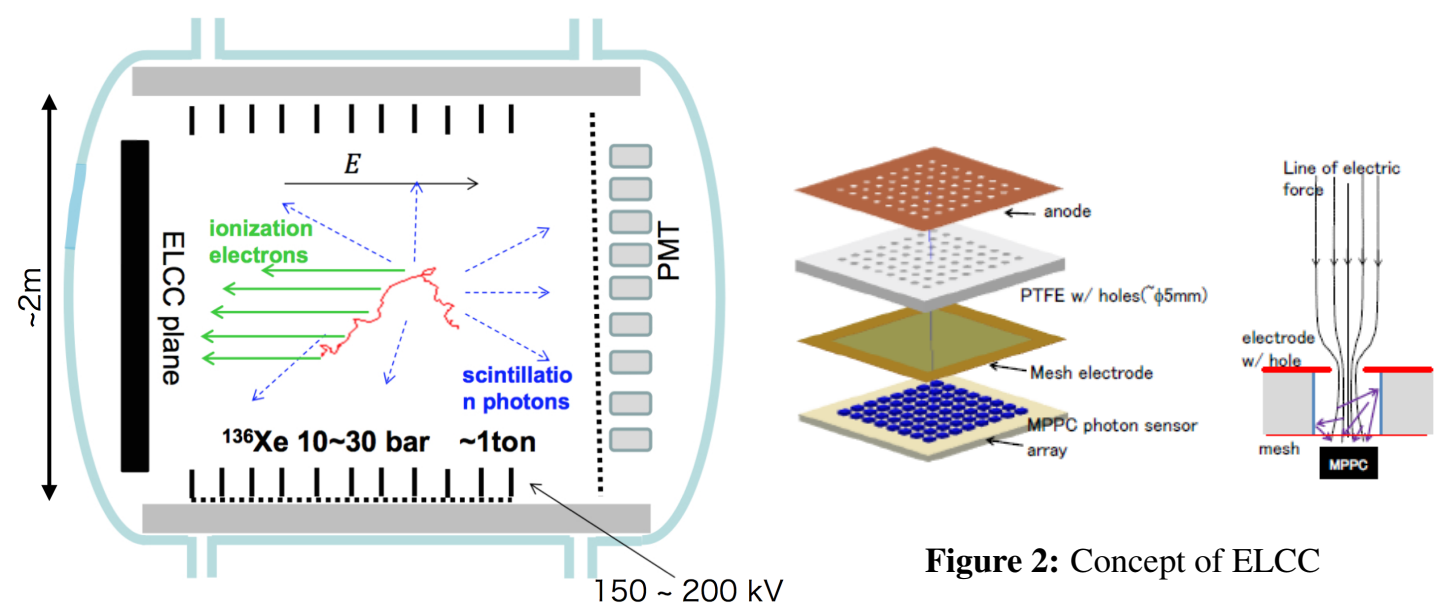

Figure 2: Concept of ELCC

Figure 1: Overview of the AXEL detector

\section{R\&D status}

\subsection{Demonstration with prototype chamber}

We have developed a small prototype detector with a $6 \mathrm{~cm}$-long and $10 \mathrm{~cm}$ diameter sensitive volume. The pitch of the cell is $7.5 \mathrm{~mm}$ and the number of cells is 32 . Performance at 4 bar Xe gas was evaluated using gamma ray from a ${ }^{57}$ Co source. Since the UV-sensitive MPPC was not available, normal MPPC with polystyrene sheet coated with wavelength shifter(TPB) was used. 


\subsubsection{Energy resolution}

Events contained in $4 \times 4$ cells were used (See Fig.[B]). Figure $\mathbb{A}$ shows the distribution of the total number of photons which corresponds to the deposited energy. Three peaks corresponding $\mathrm{K}_{\alpha} \mathrm{X}$-ray from Xenon $(29.8 \mathrm{keV})$, escape peak of $\gamma$-ray from ${ }^{57} \mathrm{Co}(92 \mathrm{keV})$ and full peak of ${ }^{57} \mathrm{Co}$ $(122 \mathrm{keV})$ are clearly seen. The energy resolution was evaluated by fitting these peaks with Gaussian. The obtained FWHM resolution is $3.4 \%, 3.4 \%$ and $2.3 \%$ for $30 \mathrm{keV}, 92 \mathrm{keV}$ and $122 \mathrm{keV}$, respectively.

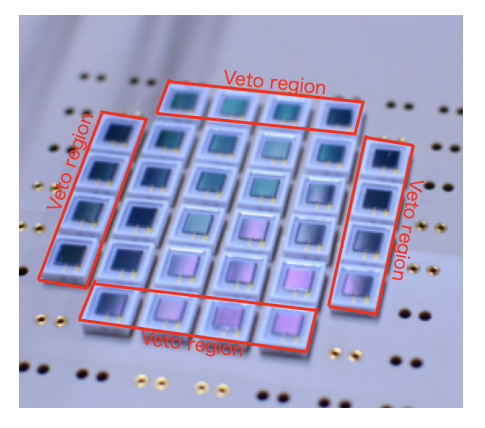

Figure 3: Configuration of MPPCs

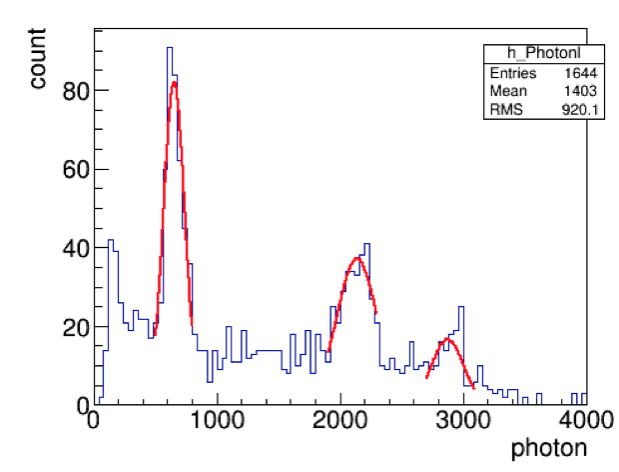

Figure 4: Distribution of the number of EL photons for ${ }^{57}$ Co source irradiation

\subsubsection{Dependence on electric field strength}

We checked the photon gain dependence on the strength of the electric field applied to drift region $\left(\mathrm{E}_{\mathrm{drift}}\right)$ and ELCC region $\left(\mathrm{E}_{\mathrm{EL}}\right)$. The dependence on $\mathrm{E}_{\mathrm{drift}}$, when $\mathrm{E}_{\mathrm{EL}}$ is fixed at $* * * \mathrm{~V} / \mathrm{cm}$ is shown in figure [1. The number of photons is decreased at higher electric field. It is because the collection efficiency of ionized electrons becomes bad, and this result is consistent to electric field calculation using finite element method. The number of photons also decreases at lower electric field. It may be because electrons are attached by ions during drift. The photon gain dependence on $\mathrm{E}_{\mathrm{EL}}$ is shown in figure 6 . The number of photons increases linearly with higher electric field and this result is qualitatively consistent with the dependence of the electroluminescence yield on the field strength[四].
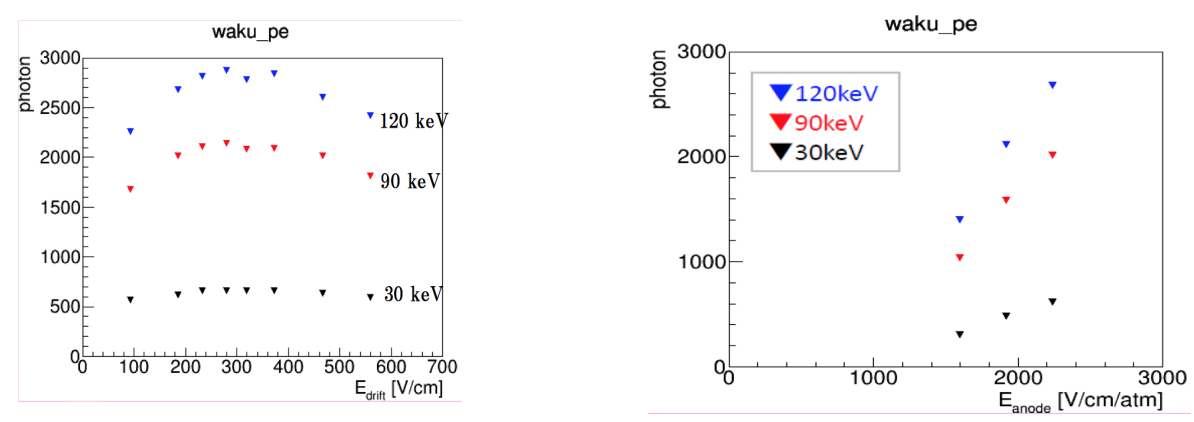

Figure 5: Number of photons as a function of Figure 6: Number of photons as a function of $\mathrm{E}_{\mathrm{drift}}$ when $\mathrm{E}_{\mathrm{EL}}$ is fixed at $8960 \mathrm{~V} / \mathrm{cm} \quad \mathrm{E}_{\mathrm{EL}}$ 


\subsection{Measurement of the basic properties of MPPC}

\subsubsection{VUV photon detection efficiency (PDE)}

The electroluminescence photon from Xe gas is pealed at VUV region (170nm). HAMAMATSU photonics recently developed MPPCs sensitive to VUV photon[[]]. We have a setup to measure the MPPC's photon detection efficiency for VUV photons from 10 bar Xe gas. VUV sensitive PMTs are used as a reference. Figure $\square$ shows scintillation light from an alpha source detected by the two PMTs. The PDE of MPPCs are now being evaluated.

\subsubsection{Liniarity}

Linearity of MPPC is very important to realize high energy resolution. In AXEL, many photons $\left(\sim 10^{5}\right)$ would be generated in relatively long time $(\sim 5 \mu \mathrm{sec})$. The linearity of MPPC for such a long pulse was examined by using LED light and by comparing with a PMT. Figure 8 shows the results of linearity measurement of two types pitch of MPPC. Good linearity was observed up to $4 \times 10^{4}$ photons $/ 5 \mu \mathrm{s}$ ( $50 \mu \mathrm{m}$-pitch, 3,600 pixels), $5 \times 10^{4}$ photons $/ 5 \mu \mathrm{s}(25 \mu \mathrm{m}$-pitch, 14,400 pixels). However it is not sufficient. In order to solve this problem, we have some options such as replacing with finer-pitch MPPC or making ELCC pitch closer to decrease the number of photons for each ELCC cell.

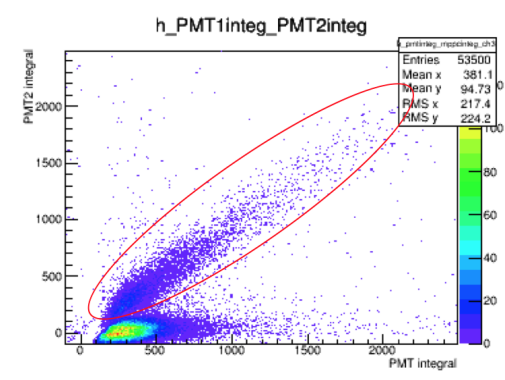

Figure 7: Scintillation light signal detected by two PMTs

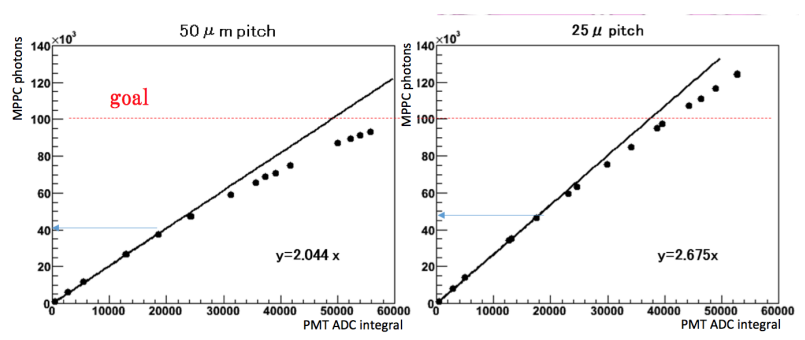

Figure 8: Linearity test of MPPC

\section{Summury}

AXEL is a $0 v \beta \beta$ search experiment with high pressure Xe gas TPC having three excellent features : high energy resolution, large mass and background rejection ability. It is now in R\&D phase. We demonstrated the concept of AXEL detector with a prototype detector and achieved the energy resolution of about 3\% FWHM for $122 \mathrm{keV}$ gamma rays. This value is not sufficient to our final goal, $0.5 \%$ FWHM, but we are now upgrading the prototype detector by increasing the number of cell to 64, installing a timing PM etc. Basic properties of MPPCs are also studied.

\section{References}

[1] Elena Aprile, Aleksey E. Bolotnikov, Alexander I. Bolozdynya and Tadayoshi Doke, Noble Gas Detector, ISBN : 978-3-527-40597-8.

[2] Kaneko, D., Performance of UV-sensitive MPPC for liquid xenon detector in MEG experiment, DOI:10.1109/NSSMIC.2013.6829484. 